\title{
Principais Direcionadores de Compra de Carnes em Hipermercados
}

\author{
Main Hypermarket Meat Purchasing Drivers
}

\section{RESUMO}

Objetivo: a urbanização e migração para os grandes centros tem provocado mudanças no comportamento dos consumidores de carnes, não somente no que se refere a escolha do produto, mas também, ao processo de compra. Neste sentido, fatores como proximidade, disponibilidade do produto, e preço são relacionados como fatores que definem compras. Na tentativa de ampliar o entendimento sobre o que os consumidores consideram importante, este trabalho objetiva analisar os principais fatores associados ao consumo das carnes bovina, suína e de frango no Distrito Federal, Brasil. Métodos: a pesquisa foi realizada em três redes distintas de hipermercados. Realizou-se uma avaliação socioeconômica dos entrevistados, seguida da aplicação de um Survey, através de sentenças afirmativas estruturadas, categorizadas em dimensões sociocultural, econômica, saúde/ alimento e ambiente. As sentenças foram avaliadas através da Escala Likert de sete pontos. Os dados socioeconômicos dos entrevistados foram analisados por testes de Qui-quadrado e as respostas obtidas por meio da Escala Likert foram transformados em Mean Item Score (MIS). Resultados e Conclusões: os resultados indicaram que o controle de qualidade de exposição das carnes nos supermercados e a praticidade de encontrar o mesmo tipo de carne em vários locais são os principais influenciadores de tomada de decisão de compra pelos consumidores.

Palavras-chave: comportamento do consumidor; preferências; compra; tomada de decisão.

\section{Classificação JEL: L8, Q21, O18.}

'Universidade de Brasília, Programa de Pós-Graduação em Agronegócios, Brasília, DF, Brasil.

${ }^{2}$ Universidade de Brasília, Campus Planaltina, Planaltina, DF, Brasil.

${ }^{3}$ Universidade de Brasília, Departamento de Estatística, Brasília, DF, Brasil.

\author{
Lierk Kalyany Silva de Sousa ${ }^{10}$ \\ Vânia Ferreira Roque-Specht ${ }^{2}{ }^{\oplus}$ \\ Eduardo Monteiro de Castro Gomes ${ }^{3}$
}

\section{ABSTRACT}

Objective: urbanization and migration to large centers has caused changes in the behavior of meat consumers, not only with regards to product choice based on product quality and safety, but also the purchasing process. In this sense, factors such as proximity, product availability, and price are also factors affecting the purchase choices. This work aims to analyze the main factors associated with the consumption of these meats in the Federal District, Brazil, in an attempt to broaden our understanding of what consumers consider important, and to provide guidance. Method: this research was carried out in three different hypermarket networks. A socioeconomic evaluation of the interviewees was carried out, followed by the application of a survey, through structured affirmative sentences that were categorized in sociocultural, economic, health/food and environmental dimensions. The sentences were evaluated using the seven-point Likert scale. Socioeconomic data pertaining the interviewees were analyzed by Chi-square tests and the responses obtained through the Likert scale were transformed into Mean Item Score (MIS). Results and Conclusions: the results indicate that the quality control of the meat exposed in supermarkets and the possibility of finding the same type of meat in several different places are the main factors influencing the purchasing decisions of consumers of these meats.

Keywords: consumer behavior; preferences; purchase; decision making.

Como citar: Sousa, L. K. S. de, Roque-Specht, V. F., \& Gomes, E. M. de C. (2020). Main hypermarket meat purchasing drivers. Revista de Administração Contemporânea, 24(4), 335-348. https://doi.org/10.1590/19827849rac2020190097

Editor-chefe: Wesley Mendes-Da-Silva (Fundação Getulio Vargas, EAESP, Brasil) Pareceristas: Miguelangelo Gianezini (Universidade do Extremo Sul Catarinense, Brasil) Um dos individuos revisores optou por não ter sua identidade divulgada.

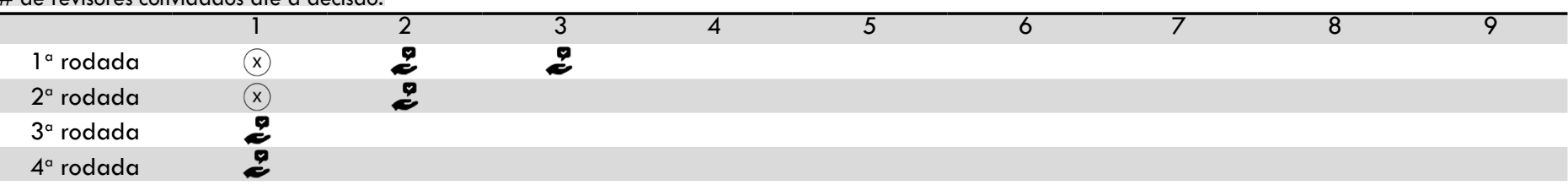




\section{INTRODUÇÃO}

O agronegócio brasileiro vem registrando importantes avanços quali-quantitativos para a economia brasileira, aumentando a capacidade empregadora e de geração de renda. O desempenho médio do agronegócio tem superado o do setor industrial, ocupando assim a posição de destaque, com importância crescente no processo de desenvolvimento econômico, principalmente pela sua capacidade de impulsionar os demais setores (Santos, Tavares, Vasconcelos, \& Afonso, 2012).

A agropecuária destaca-se na economia brasileira por meio de resultados significativos de produção de carnes e consumo, constituindo um componente básico de alimentação da sociedade. Neste sentido, projeções sobre o consumo de carnes no Brasil, no período de $2011 / 2012$ a 2021/2022, indica que a carne suína passará para a terceira colocação, com uma projeção de taxa de consumo de apenas de 1,8\% ao ano; a de frango continuará sendo a de maior preferência, com uma taxa de $2,7 \%$ ao ano e a carne bovina apresentará uma taxa de 2,0\% ao ano (Ministério da Agricultura, Pecuária e Abastecimento, 2011).

Em conjunto com a expansão populacional e as especificidades dos consumidores, o Ministério da Agricultura, Pecuária e Abastecimento do Brasil (Ministério da Agricultura, Pecuária e Abastecimento, 2011) estima um aumento na produção de carne brasileira esperado crescimento de $20,5 \%$ para carne bovina, $28,6 \%$ para a carne suína e $33,4 \%$ para a carne de frango, no período de 2017 a 2027.

Segundo Oshiiwa, Repetti, Temoteo, Labate, Pereira e Nunis (2017), as carnes são vistas pelos consumidores como alimentos nobres, de qualidade e que atendem às suas necessidades nutricionais, principalmente os que se preocupam em buscar maior expectativa de vida, com mais saúde.

Alterações na propensão dos consumidores em consumir determinadas carnes podem impactar a cadeia de produção, especialmente na determinação da quantidade demandada e ofertada. Entender os consumidores e as tendências de consumo permite o planejamento e organização de um crescimento estruturado com sustentabilidade e, em pensamento oposto, impedir que matérias-primas, insumos e energia sejam gastos de maneira desnecessária (Horvat, Granato, Fogliano, \& Luning, 2019; Zylberstajn \& Neves, 2000). Entretanto, para estudar um determinado tipo de mercado do agronegócio, conhecer e analisar seus consumidores, deve-se buscar informações de como os produtos são utilizados e como são valorados pelos consumidores. Também é necessário analisar a frequência de uso desses produtos, assim como os hábitos de compra dos consumidores em relação ao local de venda. Em consonância ao comportamento do consumidor, a aquisição de um produto ou serviço transcorre de estímulos do sujeito aos bens e serviços a sua disposição. Uma empresa que busca a competitividade deve observar as dimensões que impressionam e inspiram seus consumidores na tomada de decisão no ato da obtenção de um determinado produto (Mazzachetti \& Batalha, 2004).

Saber como pensa o consumidor e sua frequência de uso de um produto, assim como o uso e os hábitos de compra em relação ao local e a satisfação do produto adquirido é de grande relevância para se estudar o mercado (Farm, 2017). Em relação a um produto ou a um serviço colocados à disposição do consumidor, devese levar em conta o conhecimento do comportamento humano para levá-lo a uma motivação de compra (Brown, Viriyavipart, \& Wang, 2018).

Em um cenário de mercado, destaca-se a relevância do varejo no contexto mercadológico e econômico e de como ele afeta a todos de forma direta e indireta. O varejo é o principal elemento de ligação com os canais de distribuição, é também importante elemento do marketing, que permite explorar a utilidade de posse, o tempo e o lugar, buscando praticidade e rapidez no atendimento e na entrega (Las Casas \& Garcia, 2007; Martinez, Rodriguez, Mercurio, Bragg, \& Elbel, 2018).

Apesar da região Centro-Oeste se destacar na produção, em unidades de animais, de bovinos (59.609.744), suínos (7.083.205) e frangos (206.633.506), o Distrito Federal (DF), em comparação com os demais estados brasileiros, apresenta produção discreta, 63.009, 154.257, 12.171.977, respectivamente. Entretanto, o DF apresenta a maior densidade demográfica do Brasil, com 444,66 habitantes $/ \mathrm{km}^{2}$. Além disso, o DF destacase por sua população economicamente ativa apresentar a maior renda per capita do país (Instituto Brasileiro de Geografia e Estatística [IBGE], 2019). Nesse contexto, apesar do DF não ser grande produtor de carne bovina, suína e de frango, apresenta um dos maiores mercados consumidores do país.

Apesar de existirem estudos de consumo sobre o consumo de carnes em outras regiões do Brasil (Brandão, 2013), este trabalho é o primeiro a abordar o assunto no DF, contemplando simultaneamente as carnes bovinas, suínas e de frango. Para tanto, pesquisouse a prática do consumo das carnes e os desafios de atender a satisfação dos consumidores, proporcionando subsídios para traçar estratégias para futuras ações que poderão ser desenvolvidas para novos produtos e para o mercado varejista. Dessa forma, objetivou-se identificar e avaliar os direcionadores de consumo de carne (bovina, suína e aves) no comércio varejista do Distrito Federal, promovendo uma melhor avaliação do processo de compra e o conhecimento aprofundado de questões de maior relevância na escolha das carnes pelos consumidores. 


\section{Preferências do consumidor}

Considerando a imensa variedade de bens e serviços disponíveis no mercado e sua diversidade de gostos pessoais, o termo Cesta de Mercado consiste em uma lista com quantidades distintas de um ou mais bens com quantidades específicas de uma ou mais mercadorias. As preferências dos consumidores também podem ser apresentadas por Curvas de Indiferença que representa todas as combinações de cestas de mercado que fornecem o mesmo nível de satisfação a uma pessoa, para a qual, portanto, são indiferentes as cestas de mercado representadas ao longo da curva (Pindyck \& Rubinfeld, 2012).

Segundo Aguiais e Figueiredo (2015), oferecer produtos diferenciados no quesito informações sobre a carne e seu preço tem importância relevante na percepção de qualidade e preferência dos consumidores, principalmente quanto à sua apresentação. Por conseguinte, a informação está amarrada a uma escolha que tem riscos e perspectivas dos consumidores, com impacto no mercado (Stiglitz \& Walsh, 2003). Pindyck e Rubinfeld (2012) descrevem que os quesitos determinantes da busca individual são: preço do bem; renda do consumidor; preço de bens substitutos e preferências individuais. Para Medeiros e Cruz (2006) e Mazaheri, Richard, Laroche e Ueltschy (2014) todos os aspectos gerais da realidade social têm uma definição do termo Cultura e o hábito de consumo no presente e no futuro interfere no convívio familiar.

Shirai (2017) evidenciou que a percepção de preço é um dos fatores determinantes significativos no consumo e, portanto, de decisões de compra. É importante que todos os envolvidos na cadeia produtiva de um produto conheçam os atributos de qualidade para favorecer os consumidores que buscam tais informações. À medida que o consumidor reconhece o padrão de qualidade e quando é divulgado nos meios de comunicação, o produto ganha confiança (AschemannWitzel, Giménez, \& Ares, 2018; Santos et al., 2012).

\section{Comportamento do consumidor}

A teoria com o enfoque no comportamento do consumidor, com viés econômico, compreende um progresso dos processos envolvidos, quando se seleciona uma compra de produtos e/ou serviços ou experiências para atender às suas vontades. A atitude em relação ao ato da compra se concentra nas consequências percebidas, revelando o que o consumidor sente ao comprar um produto, que muitas vezes influencia a sua relação com o produto em si (Solomon, 2016).

Babutsidze (2012) procura entender como os consumidores fazem suas escolhas e, segundo ele, as decisões podem ser influenciadas por dimensões internas (personalidade) e externas (meio social). Os tipos de escolhas são compreendidos por duas dimensões: (a) a experiência do consumidor com a escolha em questão (pouca/muita experiência) e (b) a dificuldade da escolha (fácil/difícil), diferenciando-se, dessa forma as escolhas, conforme a vivência dos consumidores.

A teoria do comportamento do consumidor num determinado processo de compra é extremamente complexa e envolve conhecimentos interdisciplinares relacionados às suas preferências na compra, percepção de valores, personalidade, estilo de vida. Além disso, envolve a busca de fidelidade ao produto e análise de questões socioeconômicas (Polizei, 2011). Quando um consumidor está diante de uma escolha fácil e de baixo envolvimento, é grande a possibilidade de usar a aparência do produto para decidir, sem grande esforço (Babutsidze, 2012).

Consumidor é a pessoa que têm uma necessidade ou desejo, realiza uma compra e depois descarta o produto ao longo do processo de consumo. Entretanto, o comprador e o usuário de um produto podem não ser a mesma pessoa, como quando um pai ou uma mãe escolhem roupas para um filho adolescente (escolhas que na visão do adolescente podem representar uma gafe em termos de moda), em outros casos, outra pessoa pode agir como influenciador, fazendo recomendações a favor ou contra determinados produtos, mesmo se o produto não for comprado ou usado por ele (Rachmi, Hunter, Li, \& Baur, 2018; Solomon, 2016).

Os consumidores não escolhem um bem de consumo ou serviço por um único aspecto, mas por um conjunto de razões (Louro, 2000; Rahnama \& Rajabpour, 2017). A qualidade de um produto é percebida pelos consumidores pelas funcionalidades ou serviços, porém não pode ser determinada de forma objetiva por se tratar de percepção individual.

Segundo a teoria do consumidor, as principais dimensões relacionadas à aquisição de um bem ou serviço incluem a melhor alocação de recursos, onde o consumidor escolhe seus produtos conforme suas preferências, levando em conta a sua renda maximizando sua utilidade. No entanto, outras dimensões podem estar relacionadas na escolha de alguns produtos em particular (Pindyck \& Rubinfeld, 2012). Brandão, Barcellos, Waquil, Oliveira, Gianezini e Dias (2015) observaram que a aquisição de carne bovina pelos consumidores é influenciada também pela religião, poder aquisitivo, preço, preocupação com apresentação, entre outras questões, relacionadas a quatro dimensões principais.

Cabe destacar, aspectos sociais e culturais podem exercer influência no consumo de certos tipos de proteína animal, como a carne suína, que não possui tanta representatividade no mercado de carnes, quando comparada a carne bovina e de frango. Entretanto, a carne suína se comporta como um bem substituto e complementar diante de variações no mercado de carnes, desde alterações de preços ou como dimensões 
exógenas, que impactam na satisfação e bem-estar de seus consumidores (Montanari, 2008).

Paladini (2008) busca identificar o que é relevante para o consumidor como características específicas, processo de fabricação e uma variedade de formas de apresentação. Neste mesmo sentido, a produção e o consumo de alimentos são determinados por elementos de natureza social, econômica, tecnológica, institucional, cultural e nutricional.

\section{PROCEDIMENTOS METODOLÓGICOS}

\section{Coleta de dados}

A coleta de dados foi realizada em três redes distintas de hipermercados do Distrito Federal, Brasil, de forma exploratória, não probabilística.

Com base em Park e Jung (2009), para determinar o tamanho da amostra é necessário especificar um nível de confiança, $(1-\alpha)$, um erro tolerável relativo, $D$, um número de itens, $\mathrm{k}$, usado para escala de Likert, um coeficiente de variação de uma população, $C$ e um coeficiente de correlação pareado, $\rho$. Considerando $\mathrm{D}=0,5, \mathrm{C}=0,5, \rho$ $=0,5$ e a escala Likert de 7 pontos, seria necessário 220 entrevistas. No estudo foram realizadas 220 entrevistas, entretanto, três foram desconsideradas devido aos entrevistados assinalarem a mesma nota para todas as questões.

Primeiramente, realizou-se uma avaliação socioeconômica dos entrevistados, seguido da aplicação do Survey, através de sentenças afirmativas estruturadas, na qual os consumidores avaliaram as sentenças através da escala Likert de sete pontos, sendo: 1 - discordo plenamente; 2 - discordo muito; 3 - discordo ligeiramente; 4 - indiferente; 5 - concordo ligeiramente; 6 - concordo muito; 7 - concordo plenamente. A escolha dos consumidores foi realizada por conveniência e a participação na realização da entrevista foi voluntária, sem uso de incentivos. A única condição para a participação era que os consumidores fossem maiores de 18 anos.

As dimensões analisadas no Survey basearam-se no modelo conceitual de Brandão et al. (2015), sociocultural, econômica, saúde/alimento e ambiente, extrapolando as observações para os consumidores de carnes bovina, suína e aves.

As dimensões e as respectivas sentenças afirmativas utilizadas no Survey estão descritas e codificadas na Figura 1.

\begin{tabular}{|c|c|}
\hline Dimensão & Sentença \\
\hline A - Sociocultural & $\begin{array}{l}\text { 1. Você compra este tipo de carne porque consegue achar em vários supermercados; } \\
\text { 2. Você compra este tipo de carne porque o tamanho do corte é adequado ao seu consumo; } \\
\text { 3. Você compra este tipo de carne (ou seus derivados congelados e pré-prontos) porque o seu preparo é mais rápido e fácil; } \\
\text { 4. Você compra este tipo de carne (ou seus derivados congelados e pré-prontos) porque você trabalha fora e não tem muito } \\
\text { tempo de cozinhar; } \\
\text { 5. Você compra este tipo de carne porque é mais adequada para as atividades de sua profissão; } \\
\text { 6. Você compra este tipo de carne porque a sua religião permite; } \\
\text { 7. Você compra este tipo de carne porque é tradição na sua família; } \\
\text { 8. Você compra este tipo de carne porque a família influencia na escolha da compra; } \\
\text { 9. Você compra este tipo de carne porque você estudou sobre as suas características nutricionais }\end{array}$ \\
\hline B - Econômica & $\begin{array}{l}\text { 10. Você compra este tipo de carne porque é mais barato do que os outros tipos de carne; } \\
\text { 11. Você compra este tipo de carne porque os "substitutos de carne", por exemplo, as leguminosas (soja, feijão, grão de } \\
\text { bico...) são mais caros; } \\
\text { 12. Você compra este tipo de carne, porque as frutas e vegetais são mais caros; } \\
\text { 13. Você compra este tipo de carne porque sua renda permite comprar apenas este tipo de carne; } \\
\text { 14. Você compra este tipo de carne porque o supermercado normalmente apresenta ofertas deste produto; } \\
\text { 15. Você compra esta carne porque os substitutos em geral, são mais caros; } \\
\text { 16. Você compra este tipo de carne porque a sua renda familiar tem melhorado; } \\
\text { 17. Você compra este tipo de carne porque os preços nos mercados estão muito altos; } \\
\text { 18. Você compra este tipo de carne porque ela é exportada. }\end{array}$ \\
\hline C - Saúde/ alimento & $\begin{array}{l}\text { 19. Você compra este tipo de carne porque possui um controle de qualidade melhor na criação dos animais; } \\
\text { 20. Você compra este tipo de carne porque possui um controle de qualidade melhor nos abatedouros e frigoríficos; } \\
\text { 21. Você compra este tipo de carne porque apresenta controle de qualidade na exposição para as vendas; } \\
\text { 22. Você compra este tipo de carne porque essa carne não transmite doenças às pessoas que consomem; } \\
\text { 23. Você compra este tipo de carne porque ela promove mais saúde para o seu organismo. }\end{array}$ \\
\hline D - Ambiente & $\begin{array}{l}\text { 24. Você compra este tipo de carne porque a sua produção não promove o aumento de emissão de gases (mudanças } \\
\text { climáticas do nosso planeta); } \\
\text { 25. Você compra este tipo de carne porque a sua produção não provoca o desmatamento florestal; } \\
\text { 26. Você compra este tipo de carne porque a sua produção não afeta o abastecimento de água na cidade; } \\
\text { 27. Você compra este tipo de carne porque a sua produção atende a legislação ambiental. }\end{array}$ \\
\hline
\end{tabular}

Figura 1. Dimensões e respectivas sentenças afirmativas utilizadas no Survey. 


\section{Validação do survey}

Após a conclusão da elaboração das sentenças, o Survey foi avaliado por 12 especialistas, das áreas de Marketing, Estatística e Administração de Empresas e as observações foram consideradas no Survey.

Um pré-teste foi realizado em três hipermercados, utilizando uma amostra de 17 respondentes. Os resultados foram tabulados e validados através do coeficiente Alfa de Cronbach (Cronbach, 1951). Segundo CampoArias e Oviedo (2008), para o questionário ser aceitável, o valor mínimo para o alfa deve ser 0,70 . Abaixo desse valor, a consistência interna da escala utilizada é baixa e as questões do questionário devem ser revisadas. Neste estudo, o teste de Alfa de Cronbach, indicou os seguintes coeficientes para as dimensões: Sociocultural: 0,7021; Econômico: 0,7731; Saúde/Alimento: 0,8636 e Ambiente: 0,9155. Como todos os valores cumpriram o requisito mínimo do teste Alfa de Cronbach, prosseguiu-se com a aplicação do questionário.

\section{Procedimento de análises}

Os dados socioeconômicos dos entrevistados foram analisados por associação entre diferentes variáveis qualitativas, por meio de testes de Qui-quadrado, após o teste de normalidade de Shapiro-Wilk ter demonstrado a ausência de normalidade dos dados (Miot, 2017). O teste Qui-quadrado é utilizado para analisar associação entre diferentes variáveis qualitativas. O princípio básico deste método é comparar proporções, isto é, as possíveis divergências entre as frequências observadas na amostra e as frequências que seriam esperadas sob hipótese de independência (Levene, 1960).

Os resultados obtidos por meio da escala Likert de sete pontos foram transformados em Mean Item Score (MIS) para cada uma das declarações obtidas nas sentenças afirmativas. O MIS tem objetivo de mensurar e priorizar as dimensões e sentenças mais relevantes definidas pelos consumidores. O Mean Item Score (MIS) foi derivado da Equação 1 (Aigbavboa, Thwala, \& Eke, 2014; Mashwama, Aigbavboa, \& Thwala, 2016).

$$
\text { MIS }=(1 n 1+2 n 2+3 n 3+4 n 4+5 n 5+6 n 6+7 n 7) / N
$$

Onde:

n1 - número de respondentes de discordo plenamente;

n2 - número de respondentes de discordo muito;

n3 - número de respondentes de discordo ligeiramente;

n4 - número de respondentes de indiferente;

n5 - número de respondentes de concordo ligeiramente;

n 6 - número de respondentes de concordo muito;

n7 - número de respondentes de concordo plenamente.

$\mathrm{N}=$ total de entrevistados.

As correlações entre as dimensões sociocultural, econômica, saúde/alimento e ambiente foram analisadas pelo coeficiente de Pearson $(\rho)$, devido o teste de normalidade de Shapiro-Wilk demonstrar ausência de normalidade dos dados.

O coeficiente de Pearson $(\rho)$ varia de -1 a 1. Se as duas variáveis estiverem em perfeita relação linear, o coeficiente de correlação será 1 ou -1 . O sinal depende se as variáveis estão relacionadas positiva ou diretamente (inversamente). Conforme o coeficiente de correlação se aproxima de zero, existe uma indicação de independência ou relação não linear entre as variáveis. Além disso, os valores de $\mathrm{P}$ foram calculados para aceitar (rejeitar) a significância estatística da correlação entre essas duas variáveis considerando um nível de significância alfa de 0,05 (Ruigar \& Golian, 2015).

\section{APRESENTAÇÃO E DISCUSSÃO DOS RESULTADOS}

Os dados dos questionários foram registrados e organizados em planilhas do software Microsoft Excel, versão 2016 e no Programa Linguagem $\mathrm{R}$, versão 3.4.4, para posteriores análises.

Foram entrevistadas 217 pessoas cujos parâmetros socioeconômicos encontram-se descritos na Tabela 1. 
Tabela 1. Avaliação socioeconômicos dos consumidores de carne bovina, suína e de aves entrevistados no Distrito Federal.

\begin{tabular}{|c|c|c|c|}
\hline Variável avaliada & Parâmetro & (número de indivíduos) & $\begin{array}{c}\% \\
\text { (percentual) }\end{array}$ \\
\hline \multirow{2}{*}{ Sexo } & Masculino & 102 & 47,00 \\
\hline & Feminino & 115 & 53,00 \\
\hline \multirow{4}{*}{ Idade } & Abaixo de 20 anos & 09 & 4,15 \\
\hline & 20 a 40 anos & 148 & 68,20 \\
\hline & 40 a 60 anos & 53 & 24,42 \\
\hline & Acima de 60 anos & 7 & 3,23 \\
\hline \multirow{6}{*}{ Grau de instrução } & Fundamental incompleto & 13 & 5,99 \\
\hline & Fundamental completo & 38 & 17,51 \\
\hline & Médio incompleto & 64 & 29,49 \\
\hline & Médio completo & 58 & 26,73 \\
\hline & Superior incompleto & 44 & 20,28 \\
\hline & Superior completo & 0 & 0,00 \\
\hline \multirow{2}{*}{ Você tem alguma religião? } & Sim & 200 & 92,17 \\
\hline & Não & 17 & 7,83 \\
\hline \multirow{2}{*}{ Você é praticante? } & Sim & 170 & 78,34 \\
\hline & Não & 47 & 21,66 \\
\hline \multirow{5}{*}{ Renda da sua família } & Até 2 salários mínimos & 5 & 2,30 \\
\hline & De 2 a 4 salários mínimos & 164 & 75,58 \\
\hline & De 4 a 10 salários mínimos & 36 & 16,59 \\
\hline & De 10 a 20 salários mínimos & 9 & 4,15 \\
\hline & Acima de 20 salários mínimos & 3 & 1,38 \\
\hline \multirow{7}{*}{ Frequência do consumo de carnes } & 1 vez por semana & 21 & 9,68 \\
\hline & 2 vezes por semana & 129 & 59,45 \\
\hline & 3 vezes por semana & 52 & 23,96 \\
\hline & Mais de 4 vezes por semana & 14 & 6,45 \\
\hline & 1 vez a cada 15 dia & 1 & 0,46 \\
\hline & 1 vez por mês & 0 & 0,00 \\
\hline & 1 vez a cada 2 meses & 0 & 0,00 \\
\hline \multirow{3}{*}{ Tipo de carne mais consumida } & Frango & 87 & 40,09 \\
\hline & Bovina & 73 & 33,64 \\
\hline & Suína & 57 & 26,27 \\
\hline
\end{tabular}

Nota. Fonte: dados da pesquisa.

Os resultados (Tabela 1) revelaram que não houve discrepância com relação ao sexo e a idade da maioria dos entrevistados, que ficou entre 20 a 40 anos de idade. Com relação ao grau de instrução, houve uma homogeneidade nos percentuais dos entrevistados que possuem ensino médio completo, médio incompleto e superior incompleto, perfazendo um total de $76,50 \%$ da amostra total. Entretanto, não foram entrevistadas pessoas com nível superior completo. A maioria dos entrevistados seguia alguma religião, entretanto apenas 78,34\% destes confirmaram que eram praticantes. A renda da maioria dos entrevistados situou-se entre 2 e 4 salários mínimos. Com relação a frequência do consumo de carne, a maioria dos entrevistados indicou consumir carne duas vezes por semana. De modo geral, os consumidores preferiram, em ordem decrescente de consumo, carne de frango, bovina e suína. 
Tabela 2. Relacionamento entre as variáveis socioeconômicas por meio do teste Qui-quadrado ( $\left.\chi^{2}\right)$, com significância de $5 \%$.

\begin{tabular}{|c|c|c|c|c|c|c|c|}
\hline & Idade & $\begin{array}{c}\text { Grau de } \\
\text { instrução }\end{array}$ & $\begin{array}{l}\text { Você tem } \\
\text { alguma } \\
\text { religião }\end{array}$ & $\begin{array}{c}\text { Você é } \\
\text { praticante }\end{array}$ & $\begin{array}{l}\text { Renda } \\
\text { familiar }\end{array}$ & $\begin{array}{c}\text { Tipo de } \\
\text { carne mais } \\
\text { consumida }\end{array}$ & $\begin{array}{c}\text { Frequência } \\
\text { do consumo } \\
\text { de carnes }\end{array}$ \\
\hline Sexo & 5,664 ns & $9,362 \mathrm{~ns}$ & $0,584^{\mathrm{ns}}$ & $0,018^{\mathrm{ns}}$ & 0,889 ns & $2,928^{n s}$ & $8,435^{*}$ \\
\hline Idade & & $36,619 * * *$ & $3,268 \mathrm{~ns}$ & $0,307 \mathrm{~ns}$ & $15,797 * * *$ & $1,947 \mathrm{~ns}$ & $2,752 \mathrm{~ns}$ \\
\hline $\begin{array}{l}\text { Grau de } \\
\text { instrução }\end{array}$ & & & $13,658 * *$ & $29,844 * * *$ & $80,643 * * *$ & $48,073 * * *$ & $36,169 * *$ \\
\hline $\begin{array}{l}\text { Você tem } \\
\text { alguma } \\
\text { religião? }\end{array}$ & & & & $61,801 * * *$ & $3,985 \mathrm{~ns}$ & $1,373^{\mathrm{ns}}$ & $1,496 \mathrm{~ns}$ \\
\hline $\begin{array}{l}\text { Você é } \\
\text { praticante? }\end{array}$ & & & & & $7,898 *$ & $12,324 * *$ & $5,007^{\mathrm{ns}}$ \\
\hline Renda familiar & & & & & & $14,850 * *$ & $32,077 * * *$ \\
\hline $\begin{array}{l}\text { Tipo de } \\
\text { carne mais } \\
\text { consumida }\end{array}$ & & & & & & & $39,675 * * *$ \\
\hline
\end{tabular}

Nota. ns- não significativo ( $p>0,05)$; * significativo $(\mathrm{p}<0,05)$; ** significativo $(\mathrm{p}<0,01)$; *** significativo $(\mathrm{p}<0,001)$. Fonte: dados da pesquisa.

Segundo a Tabela 2, a variável Idade relacionou-se positivamente com o Grau de Instrução e com a Renda Familiar. Demonstrando que os entrevistados mais velhos apresentam maior grau de instrução e renda familiar. Houve ainda relação positiva entre Grau de Instrução e Você é Praticante, Renda Familiar, Tipo de Carne Mais Consumida e Frequência do Consumo de Carnes. Dessa forma, comprovou-se haver uma relação positiva e significativa entre o grau de instrução dos entrevistados que estavam concluindo ou que concluíram o ensino médio e superior, com uma maior renda familiar, uma maior distinção na escolha do tipo de carne e maior frequência de consumo de carne.

As variáveis Você tem alguma religião?; e Você é praticante?; mostraram-se intimamente relacionadas (Tabela 2). Nesse sentido, Mokhlis (2006) e Heiman, Gordon e Zilberman (2019) descrevem que pessoas com conduta religiosa avaliam o mundo por meio de princípios de suas crenças, integrando aspectos de sua religião no seu cotidiano.

A variável Renda Familiar (Tabela 1) relacionou-se positivamente com Frequência do Consumo de Carnes, demonstrando que a disponibilidade orçamentária influencia a frequência de consumo de carnes. Neste sentido, Daniel, Cross, Koebnick e Sinha (2011) e Milford,
Mouël, Bodirsky e Rolinski (2019) apontam uma relação positiva entre demanda por carne nos países em desenvolvimento, cujo crescimento da produção e consumo é diretamente proporcional ao aumento da renda.

Os resultados da análise Mean Item Score (MIS), dos diversos tipos de carne, obtidos por meio das respostas dos consumidores no Survey, encontram-se descritos na Tabela 3 que categoriza as respostas das questões do questionário nos quesitos: alta importância; média importância, baixa importância e sem importância.

A categorização usando MIS visa avaliar o quanto o consumidor considera cada quesito para a escolha do tipo de carne. Cabe salientar que não existe uma orientação de como classificar os valores de MIS. Desta forma, neste estudo, convencionou-se a utilização dos valores de MIS para as categorias: extrema importância (score igual e acima de 6,0), muito importante (score de 5,0 a 5,9 ), regular importância (score de 4,0 a 4,9 ), pouco importante (score de 2,0 a 3,9) e sem importância (score menor de 2,0). 
Tabela 3. Categorias de importância das dimensões e das sentenças utilizadas no Survey em função dos valores de Mean Item Score (MIS), para os diversos tipos de carne.

\begin{tabular}{|c|c|c|c|c|c|c|c|c|}
\hline \multirow[t]{2}{*}{$\begin{array}{l}\text { CATEGORIA DE } \\
\text { IMPORTÂNCIA }\end{array}$} & \multicolumn{2}{|c|}{ TODAS AS CARNES } & \multicolumn{2}{|c|}{ CARNE BOVINA } & \multicolumn{2}{|c|}{ CARNE SUÍNA } & \multicolumn{2}{|c|}{ CARNE DE FRANGO } \\
\hline & $\begin{array}{c}\text { Dimensão } / \mathrm{n}^{\circ} \\
\text { da sentença } \\
\text { afirmativa } \\
\text { utilizada no } \\
\text { Survey }\end{array}$ & MIS & $\begin{array}{c}\text { Dimensão } / n^{\circ} \\
\text { da sentença } \\
\text { afirmativa } \\
\text { utilizada no } \\
\text { Survey }\end{array}$ & MIS & $\begin{array}{c}\text { Dimensão } / n^{\circ} \\
\text { da sentença } \\
\text { afirmativa } \\
\text { utilizada no } \\
\text { Survey }\end{array}$ & MIS & $\begin{array}{c}\text { Dimensão } / n^{\circ} \\
\text { da sentença } \\
\text { afirmativa } \\
\text { utilizada no } \\
\text { Survey }\end{array}$ & MIS \\
\hline \multirow{2}{*}{ Extrema } & $C / n^{0} 21$ & 6,558 & $\mathrm{C} / \mathrm{n}^{0} 21$ & 6,577 & $\mathrm{C} / \mathrm{n}^{0} 21$ & 6,594 & $\mathrm{C} / \mathrm{n}^{0} 21$ & 6,606 \\
\hline & $\mathrm{A} / \mathrm{n}^{0} 1$ & 6,106 & $\mathrm{~A} / \mathrm{n}^{0} 1$ & 6,103 & $\mathrm{~A} / \mathrm{n}^{0} 1$ & 6,073 & $\mathrm{~A} / \mathrm{n}^{0} 1$ & 6,095 \\
\hline \multirow{2}{*}{ Muita } & $\mathrm{C} / \mathrm{n}^{0} 23$ & 5,401 & $\mathrm{C} / \mathrm{n}^{0} 23$ & 5,419 & $\mathrm{C} / \mathrm{n}^{\circ} 23$ & 5,543 & $C / n^{\circ} 23$ & 5,341 \\
\hline & $\mathrm{C} / \mathrm{n}^{\circ} 22$ & 5,249 & $\mathrm{C} / \mathrm{n}^{\circ} 22$ & 5,242 & $\mathrm{C} / \mathrm{n}^{\circ} 22$ & 5,5 & $\mathrm{C} / \mathrm{n}^{\circ} 22$ & 5,176 \\
\hline \multirow{6}{*}{ Regular } & $\mathrm{B} / \mathrm{n}^{0} 10$ & 4,793 & $\mathrm{~B} / \mathrm{n}^{\circ} 10$ & 4,786 & $\mathrm{~A} / \mathrm{n}^{0} 3$ & 4,775 & $\mathrm{~B} / \mathrm{n}^{\circ} 10$ & 4,859 \\
\hline & $\mathrm{A} / \mathrm{n}^{0} 3$ & 4,714 & $\mathrm{~A} / \mathrm{n}^{0} 3$ & 4,707 & $\mathrm{~B} / \mathrm{n}^{\circ} 10$ & 4,652 & $\mathrm{~A} / \mathrm{n}^{0} 3$ & 4,671 \\
\hline & $\mathrm{A} / \mathrm{n}^{\circ} 9$ & 4,327 & $\mathrm{~A} / \mathrm{n}^{\circ} 9$ & 4,321 & $\mathrm{~A} / \mathrm{n}^{\circ} 9$ & 4,355 & $\mathrm{~A} / \mathrm{n}^{\circ} 9$ & 4,3 \\
\hline & $\mathrm{A} / \mathrm{n}^{0} 2$ & 4,24 & $\mathrm{~A} / \mathrm{n}^{0} 2$ & 4,228 & $\mathrm{~B} / \mathrm{n}^{0} 11$ & 4,196 & $\mathrm{~A} / \mathrm{n}^{\circ} 2$ & 4,282 \\
\hline & $\mathrm{B} / \mathrm{n}^{0} 11$ & 4,217 & $\mathrm{~B} / \mathrm{n}^{\circ} 11$ & 4,205 & $\mathrm{~A} / \mathrm{n}^{0} 2$ & 4,058 & $\mathrm{~B} / \mathrm{n}^{0} 11$ & 4,235 \\
\hline & $\mathrm{D} / \mathrm{n}^{\circ} 26$ & 4,00 & $\mathrm{D} / \mathrm{n}^{\circ} 26$ & 4,00 & & & $\mathrm{D} / \mathrm{n}^{0} 27$ & 4,012 \\
\hline \multirow{17}{*}{ Pouca } & $\mathrm{D} / \mathrm{n}^{\circ} 27$ & 3,853 & $\mathrm{D} / \mathrm{n}^{\circ} 27$ & 3,847 & $\mathrm{D} / \mathrm{n}^{\circ} 26$ & 3,891 & $\mathrm{D} / \mathrm{n}^{\circ} 26$ & 3,971 \\
\hline & $\mathrm{A} / \mathrm{n}^{\circ} 8$ & 3,825 & $\mathrm{~A} / \mathrm{n}^{\circ} 8$ & 3,823 & $D / n^{\circ} 27$ & 3,841 & $\mathrm{~A} / \mathrm{n}^{\circ} 4$ & 3,812 \\
\hline & $\mathrm{A} / \mathrm{n}^{\circ} 4$ & 3,724 & $\mathrm{~A} / \mathrm{n}^{\circ} 4$ & 3,712 & $\mathrm{~A} / \mathrm{n}^{\circ} 4$ & 3,688 & $\mathrm{~A} / \mathrm{n}^{\circ} 8$ & 3,765 \\
\hline & $\mathrm{B} / \mathrm{n}^{\circ} 14$ & 3,687 & $\mathrm{~B} / \mathrm{n}^{\circ} 14$ & 3,674 & $\mathrm{~A} / \mathrm{n}^{\circ} 8$ & 3,688 & $\mathrm{~B} / \mathrm{n}^{\circ} 14$ & 3,718 \\
\hline & $\mathrm{D} / \mathrm{n}^{\circ} 24$ & 3,599 & $\mathrm{D} / \mathrm{n}^{\circ} 24$ & 3,595 & $\mathrm{D} / \mathrm{n}^{\circ} 25$ & 3,63 & $\mathrm{D} / \mathrm{n}^{\circ} 24$ & 3,612 \\
\hline & $D / n^{\circ} 25$ & 3,581 & $D / n^{\circ} 25$ & 3,577 & $\mathrm{~B} / \mathrm{n}^{\circ} 14$ & 3,464 & $\mathrm{D} / \mathrm{n}^{\circ} 25$ & 3,588 \\
\hline & $\mathrm{C} / \mathrm{n}^{\circ} 19$ & 3,452 & $\mathrm{C} / \mathrm{n}^{\circ} 19$ & 3,447 & $\mathrm{D} / \mathrm{n}^{0} 24$ & 3,42 & C/ no 19 . & 3,553 \\
\hline & $\mathrm{B} / \mathrm{n}^{\circ} 12$ & 3,171 & $\mathrm{~B} / \mathrm{n}^{\circ} 12$ & 3,163 & $\mathrm{C} / \mathrm{n}^{\circ} 19$ & 3,29 & $\mathrm{~B} / \mathrm{n}^{\circ} 17$ & 3,271 \\
\hline & $\mathrm{B} / \mathrm{n}^{0} 17$ & 3,171 & $\mathrm{~B} / \mathrm{n}^{\circ} 17$ & 3,163 & $\mathrm{~B} / \mathrm{n}^{\circ} 12$ & 3,261 & $\mathrm{C} / \mathrm{n}^{\circ} 20$ & 3,171 \\
\hline & $\mathrm{B} / \mathrm{n}^{\circ} 15$ & 3,166 & $\mathrm{~B} / \mathrm{n}^{\circ} 15$ & 3,158 & $\mathrm{~B} / \mathrm{n}^{\circ} 15$ & 3,232 & $\mathrm{~B} / \mathrm{n}^{0} 12$ & 3,165 \\
\hline & $\mathrm{C} / \mathrm{n}^{\circ} 20$ & 2,871 & $\mathrm{~B} / \mathrm{n}^{\circ} 16$ & 2,809 & $\mathrm{~B} / \mathrm{n}^{\circ} 17$ & 2,935 & $\mathrm{~B} / \mathrm{n}^{\circ} 15$ & 3,041 \\
\hline & $\mathrm{B} / \mathrm{n}^{\circ} 16$ & 2,82 & $C / n^{\circ} 20$ & 2,674 & $\mathrm{~B} / \mathrm{n}^{\circ} 16$ & 2,775 & $\mathrm{~B} / \mathrm{n}^{\circ} 16$ & 2,906 \\
\hline & $\mathrm{B} / \mathrm{n}^{\circ} 13$ & 2,429 & $\mathrm{~B} / \mathrm{n}^{0} 13$ & 2,414 & $C / n^{\circ} 20$ & 2,601 & $\mathrm{~A} / \mathrm{n}^{\circ} 5$ & 2,541 \\
\hline & $\mathrm{A} / \mathrm{n}^{\circ} 5$ & 2,415 & $\mathrm{~A} / \mathrm{n}^{\circ} 5$ & 2,395 & $\mathrm{~A} / \mathrm{n}^{\circ} 5$ & 2,341 & $\mathrm{~B} / \mathrm{n}^{0} 13$ & 2,529 \\
\hline & $\mathrm{A} / \mathrm{n}^{\circ} 6$ & 2,143 & $\mathrm{~A} / \mathrm{n}^{\circ} 6$ & 2,126 & $\mathrm{~B} / \mathrm{n}^{\circ} 13$ & 2,239 & $\mathrm{~A} / \mathrm{n}^{\circ} 6$ & 2,171 \\
\hline & & & $\mathrm{D} / \mathrm{n}^{0} 27$ & 3,847 & & & $\mathrm{D} / \mathrm{n}^{0} 27$ & 3,971 \\
\hline & & & $\mathrm{A} / \mathrm{n}^{\circ} 8$ & 3,823 & & & & \\
\hline \multirow{4}{*}{ Sem importância } & $\mathrm{A} / \mathrm{n}^{\circ} 7$ & 1,843 & $\mathrm{~B} / \mathrm{n}^{\circ} 18$ & 1,981 & $\mathrm{~B} / \mathrm{n}^{0} 18$ & 1,957 & $\mathrm{~A} / \mathrm{n}^{0} 7$ & 1,941 \\
\hline & & & $\mathrm{A} / \mathrm{n}^{0} 7$ & 1,823 & $\mathrm{~A} / \mathrm{n}^{\circ} 6$ & 1,913 & $\mathrm{~B} / \mathrm{n}^{\circ} 18$ & 1,9 \\
\hline & & & & & $\mathrm{A} / \mathrm{n}^{\circ} 7$ & 1,681 & & \\
\hline & & & & & $\mathrm{A} / \mathrm{n}^{\circ} 8$ & 1,681 & & \\
\hline
\end{tabular}

Nota. As dimensões e as respectivas sentenças afirmativas utilizadas no Survey estão descritas nos procedimentos metodológicos na Figura 1. Fonte: dados da pesquisa.

Na categoria de extrema importância (Tabela 3), destacou-se a Dimensão C: saúde/alimento - 21. Você compra este tipo de carne porque apresenta controle de qualidade na exposição para as vendas pois foi determinante para o processo de decisão de compra dos consumidores para todos os tipos de carne, separadamente ou em conjunto. Esses resultados demonstram que os consumidores dão especial importância a apresentação dos produtos nas gôndolas dos supermercados. Resultados semelhantes foram obtidos por Chandon, Hutchinson, Bradlow e 
Young (2009), ao analisarem a influência da posição de um produto na prateleira com o tempo de decisão de compra, bem como, a apresentação do produto. Nesse sentido, Louro (2000) e Ngapo, Lozano e Varela (2018) enfatizam que o consumidor escolhe um produto pela qualidade percebida durante a exposição do produto ou pelas suas funcionalidades, porém pode ser escolhido por um conjunto de razões, mas levando em conta os aspectos de qualidade. De forma complementar, segundo T. Hansen (2005), a percepção do consumidor envolve qualidade nas expectativas dos produtos ligados mais na aparência do alimento do que nas questões ligadas ao preço. Segundo Banović, Fontes, Barreira e Grunert (2012) e Papanagiotou, Tzimitra-Kalogianni e Melfou (2013) a aparência visual cria expectativa porque as características visuais são utilizadas para avaliar a qualidade dos alimentos na escolha da carne em um ponto de compra.

Ainda na categoria de extrema importância (Tabela 3) observou-se que a Dimensão A: Sociocultural - 1. Você compra este tipo de carne porque consegue achar em vários supermercados também influenciou de forma decisiva o processo de compra de carnes pelos consumidores. Segundo Machado, Santos, Albinati e Santos (2006) e A. Hansen (2018) quanto mais consumido um produto, mais ele se torna comum na dieta de uma população. Desta forma, esta questão deve ser considerada pelas empresas beneficiadoras e redes de distribuição, para atender e criar demandas aos consumidores.

Na categoria muito importante (Tabela 3) ressalta-se a predominância da Dimensão C Saúde/Alimento, por meio dos itens 23. Você compra este tipo de carne porque ela promove mais saúde para o seu organismo e 22. Você compra este tipo de carne porque essa carne não transmite doenças às pessoas que consomem. Com relação ao item 23 ressalta-se que os consumidores percebem a importância da carne para a sua saúde. Nesse sentido, McAfee et al. (2010) abordam que a carne contribui na ingestão de diferentes nutrientes e, também, é uma fonte de muitos componentes saudáveis. Mann (2018) descreve que além de vitaminas e minerais, a carne também contém vários nutrientes bioativos e antioxidantes.
Apesar de estudos (Bovalino, Charleson, \& Szoeke, 2016; Fogelholm, Kanerva, \& Männistö, 2015; Pacheco et al., 2018) indicarem a carne vermelha como percursora de doenças cardiovasculares, os consumidores deste Survey, através do item 22, não associaram o consumo de qualquer tipo de carne, com transmissão de doenças. Essa falta de associação entre o consumo de carnes com transmissão de doenças pode ser relacionada com a baixa frequência de consumo semanal relatada no presente Survey, somente $6,45 \%$ dos entrevistados (Tabela 1) declararam consumir carne mais de quatro vezes por semana e $59,45 \%$ declararam consumir carne duas vezes por semana. Esta última proporção encontra-se dentro do recomendável para que a população não desenvolva nenhum tipo de doença, em função do consumo de carnes (Bovalino et al., 2016; Fogelholm et al., 2015; Pacheco et al., 2018).

Com relação a categoria regular, prevaleceram as Dimensões A - Sociocultural e B - Econômica (Tabela 3), em todos os tipos de carne, carne bovina, suína e de frango.

Associam-se as respostas dos consumidores às semelhanças das táticas de vendas adotadas pelos hipermercados. Shirai (2017) e Merlino, Borra, Girgenti, Vecchio e Massaglia (2018) relatam que a percepção de preço é um dos determinantes significativos nas decisões de compra do consumidor, mas esta percepção é influenciada pelas táticas desenvolvidas por fabricantes e varejistas para criar percepções de preços mais favoráveis por parte dos consumidores. Se as redes varejistas apresentarem comportamento de vendas semelhantes, a percepção de preço pelos consumidores será semelhante. A escolha da carne, em função do preparo mais rápido e fácil, pode ser relacionada com a mudança de hábito da população brasileira, em geral. Homens e mulheres trabalham fora e buscam, de alguma forma, facilitar o preparo de suas refeições. Os resultados da pesquisa de Oliveira et al. (2015, 2017) indicam que a escolha de um determinado alimento também está relacionada com a praticidade de preparo, além da preocupação com a saúde e a rotina do dia a dia. 
Tabela 4. Correlação de Pearson e significância comparando valores da escala de Likert, entre as dimensões, relacionando o consumo de carnes.

\begin{tabular}{|c|c|c|c|}
\hline & Dimensão A - Sociocultural & Dimensão B - Econômico & Dimensão C - Saúde/Alimento \\
\hline \multicolumn{4}{|c|}{ Considerando todos os tipos de carne } \\
\hline Dimensão B - Econômico & $0,580 * * *$ & & \\
\hline Dimensão C - Saúde/Alimento & $0,033 \mathrm{~ns}$ & $0,039 \mathrm{~ns}$ & \\
\hline Dimensão D - Ambiente & $0,147 *$ & $-0,007 \mathrm{~ns}$ & $0,184 * *$ \\
\hline \multicolumn{4}{|c|}{ Considerando somente carne bovina } \\
\hline Dimensão B - Econômico & $0,643 * * *$ & & \\
\hline Dimensão C - Saúde/Alimento & $0,215^{\mathrm{ns}}$ & 0,049 ns & \\
\hline Dimensão D - Ambiente & $0,210^{\mathrm{ns}}$ & 0,096 ns & $0,066^{\mathrm{ns}}$ \\
\hline \multicolumn{4}{|c|}{ Considerando somente carne suína } \\
\hline Dimensão B - Econômico & 0,173 ns & & \\
\hline Dimensão C - Saúde/Alimento & $-0,033^{\text {ns }}$ & $0,290 *$ & \\
\hline Dimensão D - Ambiente & $0,072 \mathrm{~ns}$ & $-0,020^{\mathrm{ns}}$ & $0,066^{\mathrm{ns}}$ \\
\hline \multicolumn{4}{|c|}{ Considerando somente carne de frango } \\
\hline Dimensão B - Econômico & $0,641 * * *$ & & \\
\hline Dimensão C - Saúde/Alimento & $-0,227 *$ & $-0,017^{n s}$ & \\
\hline Dimensão D - Ambiente & $0,104^{\mathrm{ns}}$ & $-0,111^{\mathrm{ns}}$ & $0,329 * *$ \\
\hline
\end{tabular}

Nota. ${ }^{\text {ns }}$ - não significativo ( $\left.>0,05\right)$; * significativo $(p<0,05)$; ** significativo $(p<0,01)$; *** significativo $(p<0,001)$.

Houve correlações positivas elevadas e significativas (Tabela 4) entre as dimensões A Sociocultural e B - Econômica, para a variável todos os tipos de carne, para a carne bovina e para carne de frango. Isso indica uma forte associação entre preço do produto com as questões socioculturais. Essa correlação significativa pode ser associada aos hábitos de vida contemporânea da população economicamente ativa, cujas atividades profissionais determinam um tempo menor para a preparação de refeições (Oliveira, Ferreira, Santana, Santos, Brito, \& Mendes, 2015; Oliveira et al., 2017). Apesar desta associação não ter sido significativa para carne suína, atribui-se ao fato de ser a carne menos consumida entre os entrevistados (Tabela 1).

As dimensões C - Saúde/Alimento e D Ambiente também se correlacionaram de forma positiva e significativa para a carne de frango e todos os tipos de carne (Tabela 4). A associação entre estas duas dimensões pode ser atribuída aos consumidores estarem cada vez mais atentos aos efeitos dos abatedouros no meio ambiente e estão exigindo controle das autoridades competentes. Isto significa que pessoas com maior desenvolvimento sociocultural tem maior discernimento para associar os efeitos negativos sobre o meio-ambiente com a sua saúde. Nesse sentido, diversos indicadores de desempenho ambiental podem auxiliar nesse controle. Entre os indicadores mais utilizados, destacam-se: consumo de água, consumo de energia, produção de alimentos, uso de produtos químicos e materiais de embalagem, descarga de águas residuais e tratamento de resíduos (Skunca, Tomasevic, Nastasijevic, Tomovic, \& Djekic, 2018).

As diferenças na proporção da ingestão de diferentes tipos de carne são, em grande parte, resultado de desigualdades econômicas, mas também refletem diferenças na eficiência de produção. No mundo industrializado, os principais avanços na criação, nutrição e práticas de criação aumentaram muito a eficiência e reduziram o custo da produção animal. Isto é particularmente verdadeiro na produção de frango e de suínos, onde uma combinação de genética, juntamente com a formulação de dietas de alta qualidade e melhores técnicas de manejo, melhorou drasticamente a eficiência da produção e tornou o preço competitivo (Salter, 2018). Essa associação foi percebida no presente estudo por meio da correlação entre as dimensões B - Econômica e C - Saúde/Alimento para a carne suína (Tabela 4).

Latvala et al. (2012) descrevem que a mudança de consumo de carnes está relacionada a várias dimensões, destacando mudanças sociodemográficas dos consumidores, questões 
como sabor, a segurança, a salubridade e o preço, mudanças nos padrões de consumo, os consumidores estão preferindo carnes mais magras e sem hormônios, e o bem-estar animal. Adicionalmente, Graça, Calheiros e Oliveira (2016) enfatizam mudanças no consumo de carnes em função de mudanças dos mecanismos cognitivos que são alterados ao longo da vida. Finalmente, Pohjolainen, Tapio, Vinnari, Jokinen e Räsänen (2016) descrevem mudanças do comportamento do consumo de carne em virtude do aumento da consciência ambiental dos consumidores e conscientização do problema. Assim, a relação inversa encontrada no presente estudo, entre as dimensões A - Sociocultural e C - Saúde/Alimento para carne de frango (Tabela 4) pode ser, pelo menos, em parte atribuída à influência isolada ou conjunta de todos estes parâmetros descritos sobre as mudanças de comportamento de consumo de carne, possivelmente omitidos nas perguntas relacionadas aos parâmetros socioculturais.

\section{CONSIDERAÇÕES FINAIS}

Essa pesquisa demonstrou que os consumidores do Distrito Federal apresentam comportamento semelhante de escolha para a compra de carne bovina, suína e de frango. Entretanto, a carne de frango foi a mais consumida, seguida da carne bovina e, por último, a carne suína.

Essa pesquisa demonstrou que apesar dos consumidores do Distrito Federal consumirem mais

\section{REFERÊNCIAS}

Aguiais, E. G., \& Figueiredo, R. S. (2015). Correlação entre consumo de carne de frango e renda no Brasil (2002-2009). Qualia: A Ciência em Movimento. 1(1), 64-77. Retrieved from https://revistas.unifan.edu.br/index.php/RevistaICSA/article/view/134

Aigbavboa, C. O., Thwala, W. D., \& Eke, C. C. (2014). An evaluation of students's satisfaction with University Hall of Residence. In D. Yang, \& Y. Qian (Eds.), Proceeding of the 18th international symposium on advancement of construction management and real estate (Chapter 68, pp. 703-711). New York: Springer.

Aschemann-Witzel, J., Giménez, A., \& Ares, G. (2018). Consumer instore choice of suboptimal food to avoid food waste: The role of food category, communication and perception of quality dimensions. Food Quality and Preference, 68, 29-39. https:// doi.org/10.1016/j.foodqual.2018.01.020 carne de frango, que bovina e suína, apresentam comportamento semelhante de escolha para a compra todos os tipos de carne.

Adicionalmente, observou-se que questões relacionadas a preços e ofertas não foram consideradas incentivadores na decisão de escolha de carnes pelos consumidores. Contudo, estes consideram a qualidade das carnes expostas nas prateleiras e a facilidade de encontrá-las em diferentes hipermercados como fatores determinantes no processo de compra. Estes resultados indicam que as empresas beneficiadoras de carnes devem criar estratégias de disponibilização de diferentes pontos de venda e de apresentação dos seus produtos. No primeiro caso, uma análise de estoque e de logística de recebimentos deve ser considerada. A apresentação dos produtos deve considerar organização, higiene, iluminação das prateleiras, como também aspectos de inocuidade e apresentação da embalagem.

Considerando-se que este estudo foi desenvolvido no Distrito Federal, que apresenta particularidades socioeconômicas específicas em relação a maioria das regiões brasileiras, para contribuir com o sistema nacional de produção e comercialização de produtos cárneos no Brasil, sugere-se que trabalhos futuros abordem o mesmo tema em outros locais.

Babutsidze, Z. (2012). How do consumers make choices? A survey of evidence. Journal of Economic Surveys, 26(4), 752-762. https://doi.org/10.1111/j.1467-6419.2011.00693.x

Banović, M., Fontes, M. A., Barreira, M. M., \& Grunert, K. G. (2012). Impact of product familiarity on beef quality perception. Agribusiness, 28(2), 157-172. https://doi.org/10.1002/agr.21290

Bovalino, S., Charleson, G., \& Szoeke, C. (2016). The impact of red and processed meat consumption on cardiovascular disease risk in women. Nutrition, 32(3), 349-354. https://doi.org/10.1016/j.nut.2015.09.015

Brandão, F. S. (2013). Tendências para o consumo de carne bovina no Brasil (Tese de doutorado). Universidade Federal do Rio Grande do Sul, Porto Alegre, RS, Brasil.

Brandão, F. S., Barcellos, J. O. J., Waquil, P. D., Oliveira, T. E., Gianezini, M., \& Dias, E. A. (2015). Conceptual model to identify factors with influence in Brazilian beef consumption. Revista Brasileira de Zootecnia, 44(6), 213-218. https://dx.doi.org/10.1590/ S1806-92902015000600003 
Brown, A. L., Viriyavipart, A., \& Wang, X. (2018). Search deterrence in experimental consumer goods markets. European Economic Review, 104, 167-184. https://doi.org/10.1016/j. euroecorev.2018.03.001

Campo-Arias, A., \& Oviedo, H. C. (2008). Psychometric properties of a scale: Internal consistency. Revista de Salud Pública, 10(5), 831-839. https://doi.org/10.1590/s012400642008000500015

Chandon, P., Hutchinson, J. W., Bradlow, E. T., \& Young, S. H. (2009). Does in-store marketing work? Effects of the number and position of shelf facings on brand attention and evaluation at the point of purchase. Journal of Marketing, 73(6), 1-17. https://doi.org/10.1509/jmkg.73.6.1

Cronbach, L. J. (1951). Coefficient alpha and the internal structure of test. Psychometrika, 16(3), 297-334. https://doi. org/10.1007/BF02310555

Daniel, C. R., Cross, A. J., Koebnick, C., \& Sinha, R. (2011). Trends in meat consumption in the USA. Public Health Nutrition, 14(4), 575-583. https://doi.org/10.1017/ S1368980010002077

Farm, A. (2017). Pricing and production in consumer markets where sales depend on production. Economics Letters, 154(1), 17-19. https://doi.org/10.1016/j.econlet.2017.02.021

Fogelholm, M., Kanerva, N., \& Männistö, S. (2015). Association between red and processed meat consumption and chronic diseases: The confounding role of other dietary factors. European Journal of Clinical Nutrition, 69, 1060-1065. https://doi.org/10.1038/ejcn.2015.63

Graça, J., Calheiros, M. M., \& Oliveira, A. (2016). Situating moral disengagement: Motivated reasoning in meat consumption and substitution. Personality and Individual Differences, 90, 353-364. https://doi.org/10.1016/j. paid.2015.11.042

Hansen, T. (2005). Understanding consumer perception of food quality: The cases of shrimps and cheese. British Food Journal, 107(7), 500-525. https://doi. org/10.1108/00070700510606909

Hansen, A. (2018). Meat consumption and capitalist development: The meatification of food provision and practice in Vietnam. Geoforum, 93, 57-68. https://doi.org/10.1016/j. geoforum.2018.05.008

Heiman, A., Gordon, B., \& Zilberman, D. (2019). Food beliefs and food supply chains: The impact of religion and religiosity in Israel. Food Policy, 83, 363-369. https://doi. org/10.1016/j.foodpol.2017.07.007

Horvat, A., Granato, G. Fogliano, V., \& Luning, P. A. (2019). Understanding consumer data use in new product development and the product life cycle in European food firms. An empirical study. Food Quality and Preference, 76, 20-32. https://doi.org/10.1016/j.foodqual.2019.03.008

Instituto Brasileiro de Geografia e Estatística. Censo demográfico 2010. Retrieved from https://cidades.ibge.gov.br/.

Las Casas, A. L., \& Garcia, M. T. (2007). Estratégias de marketing para o varejo: Inovações e diferenciações estratégicas que fazem a diferença no marketing de varejo. São Paulo: Novatec.
Latvala, T., Niva, M., Mäkelä, J., Pouta, E., Heikkilä, J., Kotro, J., \& Forsman-Hugg, S. (2012). Diversifying meat consumption patterns: Consumers' self-reported past behaviour and intentions for change. Meat Science, 92(1), 71-77. https://doi.org/10.1016/j.meatsci.2012.04.014

Levene, H. (1960). Robust tests for equality of variances. In I. Olkin, S. G. Ghurye, W. Hoeffding, W. G. Madow, \& H. B. Mann (Eds.), Contribution to probability and statistics: Essays in honor of harold hotelling (pp. 278-292). Stanford: Stanford University Press.

Louro, M. J. S. (2000). Modelos de avaliação de marca. RAE - Revista de Administração de Empresas, 40(2), 26-37. http:// dx.doi.org/10.1590/S0034-75902000000200004

Machado, S. S., Santos, F. O., Albinati, F. L., \& Santos, L. P. R. (2006). Comportamento dos consumidores com relação à leitura de rótulo de produtos alimentícios. Alimentos e Nutrição, 17(1), 97-103. Retrieved from http://serv-bib.fcfar.unesp.br/seer/index.php/alimentos/article/ viewArticle/119

Mann, N. J. (2018). A brief history of meat in the human diet and current health implications. Meat Science, 144, 169-179. https://doi.org/10.1016/j.meatsci.2018.06.008

Martinez, O., Rodriguez, N., Mercurio, A., Bragg, M., \& Elbel, B. (2018). Supermarket retailers' perspectives on healthy food retail strategies: In-depth interviews. BMC Public Health, 18. https://doi.org/10.1186/s12889-018-5917-4

Mashwama, X. N., Aigbavboa, C., \& Thwala, D. (2016). Investigation of construction stakeholders' perception on the effects \& cost of construction dispute in Swaziland. Procedia Engineering, 164, 196-205. https://doi.org/10.1016/j. proeng.2016.11.610

Mazaheri, E., Richard, M. O., Laroche, M., \& Ueltschy, L. C. (2014). The influence of culture, emotions, intangibility, and atmospheric cues on online behavior. Journal of Business Research, 67(3), 253-259. http://dx.doi.org/10.1016/j. jbusres.2013.05.011

Mazzachetti, R. N., \& Batalha, M. O. (2004). O comportamento do consumidor em relação ao consumo e às estruturas de comercialização da carne bovina na região de Amerios/ PR. Revista Varia Scientia, 4(8), 25-43. Retrieved from http://e-revista.unioeste.br/index.php/variascientia/article/view/721

McAfee, A. J., McSorley, E. M., Cuskelly, G. J., Moss, B. W., Wallace, J. M., Bonham, M. P., \& Fearon, A. M. (2010). Red meat consumption: An overview of the risks and benefits. Meat Science, 84(1), 1-13. https://doi.org/10.1016/j. meatsci.2009.08.029

Medeiros, J. F., \& Cruz, C. M. L. (2006). Comportamento do consumidor: Fatores que influenciam no processo de decisão de compra dos consumidores. Teoria e Evidência Econômica, 14(spe), 167-190. Retrieved from http://ead2.fgv. br/ls5/centro_rec/docs/comportamento_consumidor_ fatores_que_influenciam.pdf

Merlino, V. M., Borra, D., Girgenti, V., Vecchio, A. D., \& Massaglia, S. (2018). Beef meat preferences of consumers from Northwest Italy: Analysis of choice attributes. Meat Science, 143, 119-128. https://doi.org/10.1016/j.meatsci.2018.04.023 
Milford, A. B., Mouël, C. L., Bodirsky, B. L., \& Rolinski, S. (2019). Drivers of meat consumption. Appetite, 141, 104313. https://doi.org/10.1016/j.appet.2019.06.005

Ministério da Agricultura, Pecuária e Abastecimento (2011). Plano agrícola e pecuário 2011-2012. Brasília: Mapa/SPA. Retrieved from http://www.agricultura.gov.br/assuntos/politica-agricola/todas-publicacoes-de-politica-agricola/plano-agricola-pecuario/plano-agricola-e-pecuario-2011-2012.pdf/view

Miot, H. A. (2017). Avaliação da normalidade dos dados em estudos clínicos e experimentais. Jornal Vascular Brasileiro, 16(2), 88-91. https://dx.doi.org/10.1590/16775449.041117

Mokhlis, S. (2006). The effect of religiosity on shopping orientation: An exploratory study in Malaysia. The Journal of American Academy of Business, 9(1), 64-74. Retrieved from http://www.jaabc.com/jaabcv9n1preview.html

Montanari, M. (2008). Comida como cultura. São Paulo: Senac.

Ngapo, T. M., Lozano, M. S. R., \& Varela, D. B. (2018). Mexican consumers at the point of meat purchase. Pork choice. Meat Science, 135, 27-35. https://doi.org/10.1016/j. meatsci.2017.08.005

Oliveira, A. P., Ferreira, M. R., Santana, H. A. Jr., Santos, M. S., Brito, J. M., \& Mendes, F. B. L. (2015). Caracterização do consumidor de carne de frango em Júlio Borges, PI. Revista Científica de Produção Animal, 17(2), 129-141. http:// dx.doi.org/10.15528/2176-4158/rcpa.v17n2p129-141

Oliveira, A. P., Silva, C. P., Santana, H. A. Jr., Santos, M. S. dos, Brito, J. M. de, Mendes, F. B. L., \& Santana, E. O. C. (2017). Principais aspectos considerados por consumidores na aquisição e consumo de carne suína em Colônia do Piauí-PI. Arquivo de Ciências Veterinárias e Zoologia da UNIPAR, 20(2), 71-77. https://doi.org/10.25110/arqvet. v20i2.2017.5810

Oshiiwa, M., Repetti, L., Temoteo, M. M., Labate, B. Y., Pereira, A. B., \& Nunis, J. B. (2017). Perfil e atributos que influenciam na decisão de compra dos consumidores de carnes em dois supermercados de médio porte na cidade de Marília/SP. UNIMAR Ciências, 26(1-2), 95-113. Retrieved from http://ojs.unimar.br/index.php/ciencias/article/ viewFile/517/231

Pacheco, D. A. Q., Sookthai, D., Wittenbecher, C., Graf, M. E., Schübel, R., Johnson, T., Katzke, V., Jakszyn, P., Kaaks, R., \& Kühn, T. (2018). Red meat consumption and risk of cardiovascular diseases - is increased iron load a possible link? The American Journal of Clinical Nutrition, 107(1), 113-119, https://doi.org/10.1093/ajcn/nqx014

Paladini, E. P. (2008). Gestão estratégica da qualidade: Princípios, métodos e processos. São Paulo: Atlas.

Papanagiotou, P., Tzimitra-Kalogianni, I., \& Melfou, K. (2013). Consumers' expected quality and intention to purchase high quality pork meat. Meat Science, 93(3), 449-454. https:// doi.org/10.1016/j.meatsci.2012.11.024

Park, J.-W., \& Jung, M.-S. (2009). A note on determination of sample size for a Likert scale. Communications for Statistical Applications and Methods, 16(4), 669-673. https://doi. org/10.5351/CKSS.2009.16.4.669

Pindyck, R. S., \& Rubinfeld, D. L. (2012). Microeconomia (7th ed.). São Paulo: Pearson.
Pohjolainen, P., Tapio, P., Vinnari, M., Jokinen, P., \& Räsänen, P. (2016). Consumer consciousness on meat and the environment. Exploring differences. Appetite, 101, 37-45. https://doi.org/10.1016/j.appet.2016.02.012

Polizei, E. (2011). Plano de Marketing (2a ed., Rev. e amp.). São Paulo: CENGAGE Learning.

Rachmi, C. N., Hunter, C. L., Li, M., \& Baur, L. A. (2018). Food choices made by primary carers (mothers/ grandmothers) in West Java, Indonesia. Appetite, 130, 84-92, https://doi. org/10.1016/j.appet.2018.08.005

Rahnama, H., \& Rajabpour, S. (2017). Factors for consumer choice of dairy products in Iran. Appetite, 111, 46-55. http:// dx.doi.org/10.1016/j.appet.2016.12.004

Ruigar, H., \& Golian, S. (2015). Assessing the correlation between climate signals and monthly mean and extreme precipitation and discharge of Golestan Dam Watershed. Earth Sciences Research Journal, 19(1), 65-72. https://dx.doi. org/10.15446/esrj.v19n1.40996

Salter, A. M. (2018). The effects of meat consumption on global health. Revue Scientifique et Technique (International Office of Epizootics), 37(1), 47-55. http://dx.doi. org/10.20506/rst.37.1.2739

Santos, J. A. M. dos, Tavares, M. C., Vasconcelos, M. C. R. L. de, \& Afonso, T. (2012). O processo de inovação tecnológica na Embrapa e na Embrapa Agrobiologia: Desafios e perspectivas. Perspectivas em Ciência da Informação, 17(4), 175-194. https://dx.doi.org/10.1590/S141399362012000400011

Shirai, M. (2017). Effects of price reframing tactics on consumer perceptions. Journal of Retailing and Consumer Services, 34, 82-87. https://doi.org/10.1016/j.jretconser.2016.09.009

Skunca, D., Tomasevic, I., Nastasijevic, I., Tomovic, V., \& Djekic, I. (2018). Life cycle assessment of the chicken meat chain. Journal of Cleaner Production, 184, 440-450. https://doi. org/10.1016/j.jclepro.2018.02.274

Solomon, M. R. (2016). O comportamento do consumidor: Comprando, possuindo e sendo (11a. ed.) Porto Alegre: Bookman.

Stiglitz, J. E., \& Walsh, C. E. (2003). Introdução à economia. Rio de Janeiro: Campus.

Zylberstajn, D., \& Neves, M. F. (2000). Economia e gestão dos negócios agroalimentares: Indústria de alimentos, indústria de insumos, produção agropecuária e distribuição. São Paulo: Pioneira. 


\section{Autores}

\section{Lierk Kalyany Silva de Sousa}

Campus Universitário Darcy Ribeiro, s/n, 70910-900, Brasília, DF, Brasil.

E-mail: lierksousa@gmail.com

• https://orcid.org/0000-0002-6805-1264

\section{Vânia Ferreira Roque-Specht*}

Área Universitária, 01, Vila Nossa Senhora de Fátima, 73345010, Planaltina, DF, Brasil.

E-mail:vaniars@unb.br

(- https://orcid.org/0000-0002-5903-3072

\section{Eduardo Monteiro de Castro Gomes}

Campus Universitário Darcy Ribeiro, s/n, 70910-900, Brasília, DF, Brasil.

E-mail: edumonteiro@unb.br

ㄴ https://orcid.org/0000-0002-8948-9855

* Autor Correspondente

\section{Contribuições dos Autores}

$1^{\text {a }}$ autora: Projeto de pesquisa, desenvolvimento teóricometodológico, aplicação, redação e revisão final.

$2^{\mathbf{a}}$ autora: Projeto de pesquisa, desenvolvimento teóricometodológico, aplicação, redação e revisão final.

$3^{\circ}$ autor: Projeto de pesquisa, desenvolvimento teóricometodológico, análise estatística, redação e revisão final.

\section{Financiamento}

Os autores informaram que não houve apoio financeiro para a pesquisa neste artigo.

\section{Conflito de Interesses}

Os autores informaram que não há conflito de interesses.

\section{Direitos Autorais}

A RAC detém os direitos autorais deste conteúdo.

\section{Verificação de Plágio}

A RAC mantém a prática de submeter todos os documentos aprovados para publicação à verificação de plágio, mediante o emprego de ferramentas específicas, e.g.: iThenticate.

\section{Método de Revisão por Pares}

Este conteúdo foi avaliado utilizando o processo de revisão por pares duplo-cego (double-blind peer-review). A divulgação das informações dos pareceristas constantes na primeira página é feita somente após a conclusão do processo avaliativo, e com o consentimento voluntário dos respectivos pareceristas.

Disponibilidade dos Dados

Todos os dados e materiais foram disponibilizados publicamente por meio da plataforma Mendeley e podem ser acessados em:

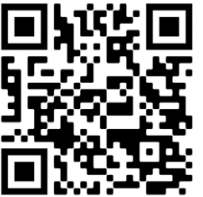

Kalyany Silva de Sousa, Lierk; Roque-Specht, Vânia; Monteiro de Castro Gomes, Eduardo (2020), “"Data for: Main hypermarket meat purchasing drivers" published by RAC-Revista de Administracão Contemporânea", Mendeley Data, v3. http://dx.doi.org/10.17632/5k53393m5c.3 